\title{
CORRELATION FUNCTIONS IN THE SPIN-FERMION MODEL
}

\author{
J. Bala AND A.M. Oleś \\ Institute of Physics, Jagellonian University, Reymonta 4, 30-059 Kraków, Poland
}

\begin{abstract}
We show that the quasiparticle states found in a self-consistent Born approximation for the spin-fermion model which describes $\mathrm{CuO}_{2}$ planes agree well with experimental data in insulating $\mathrm{Sr}_{2} \mathrm{CuO}_{2} \mathrm{Cl}_{2}$. It is found that the antiferromagnetic correlations are reduced in the neighbourhood of a moving oxygen hole and the correlation functions can exhibit similar dipolar distortions as reported in the context of the $t-J$ model.
\end{abstract}

PACS numbers: 71.27.+a, 75.10.-b, 74.72.-h, 79.60.-i

Recent angle-resolved photoemission spectroscopy (ARPES) measurements of insulating $\mathrm{Sr}_{2} \mathrm{CuO}_{2} \mathrm{Cl}_{2}[1,2]$ showed a strongly $k$-dependent quasiparticle (QP) peak, representing a coherent propagation of Zhang-Rice (ZR) singlets [3] coexisting with strongly $k$-dependent higher energy oxygen bands. These experiments stimulated theoretical investigations of the QP states in the $t-J$ model with extended hoppings [4] and using more realistic Hamiltonians [5, 6] describing the motion of a single carrier in a two-dimensional antiferromagnet. To calculate the spectral properties of the entire oxygen-like valence band we consider below a realistic charge-transfer (CT) model which takes into account $\mathrm{Cu}\left(3 d_{x^{2}-y^{2}}\right)$ and all in-plane oxygen orbitals $\mathrm{O}\left(2 p_{\sigma}, 2 p_{\pi}\right)$ playing a dominant role in $\mathrm{CuO}_{2}$ planes. The $\pi$-oxygen states (hybridized with the $\sigma$ ones) were included as they were also observed with a strong momentum dependence at higher energies in the ARPES experiment by Pothuizen et al. [2].

Recently Reiter [7] has derived a wave function for a single hole in the $t-J$ model to all orders in the number of excited magnons in the antiferromagnetic (AF) background which is exact in the self-consistent Born approximation (SCBA) [8]. With the help of the explicit form of the QP wave function corresponding to the SCBA we can calculate various correlation functions relative to the position of the oxygen hole. Such functions and the correlation functions around a hole were investigated within $t-J$ and $t-J^{z}$ models by Ramšak and Horsch [9]. Here we extend their study to the case of oxygen hole in the spin-fermion model.

In the limit of weak $\mathrm{Cu}-\mathrm{O}$ hybridization $\left(t_{p d}\right)$ as compared with the Coulomb repulsive energy at copper sites $(U)$ and the CT energy $(\Delta)$, i.e., $\left|t_{p d}\right| \ll U$ and $\left|t_{p d}\right| \ll \Delta$, we derived an effective Hamiltonian. Following the procedure described 
in more detail in Ref. [6] after Fourier and Bogoliubov transformations one finds in the linear spin-wave (LSW) order the effective Hamiltonian of the form

$$
\begin{aligned}
& H_{\mathrm{LSW}}=\sum_{\boldsymbol{k} \mu s} E_{\mu}(k) p_{\boldsymbol{k}, \mu s}^{\dagger} p_{k, \mu s}+\sum_{\boldsymbol{q}} \omega_{\boldsymbol{q}} \beta_{\boldsymbol{q}}^{\dagger} \beta_{\boldsymbol{q}} \\
& \quad+\sum_{\boldsymbol{k}, \mu \nu s} M_{\mu \nu}(k, \boldsymbol{q}) p_{\boldsymbol{k}-\boldsymbol{q}, \mu s}^{\dagger} p_{\boldsymbol{k}, \nu,-s}\left(\beta_{\boldsymbol{q}}^{\dagger}+\beta_{-\boldsymbol{q}}\right) .
\end{aligned}
$$

$E_{\mu}(k)$ are the oxygen subbands and $M_{\mu \nu}(k, q)$ is the hole-magnon vertex depending on the coefficient of the Bogoliubov transformation for bosons $\left\{u_{\boldsymbol{q}}, v_{\boldsymbol{q}}\right\}$, and for fermions $\left\{V_{i \nu}(k)\right\}$.

The Green function is determined in the SCBA by the hole self-energy obtained from the bare vertex and the self-consistently dressed Green function

$$
G_{\mu \nu}^{-1}(k, \omega)=\omega-\delta_{\mu \nu} E_{\mu}(k)-\sum_{\alpha \beta, q} M_{\mu \alpha}(k, q) M_{\beta \nu}(k, q) G_{\alpha \beta}\left(k-\boldsymbol{q}, \omega-\omega_{q}\right) .
$$

With the help of the Green function, we can obtain the Reiter wave function $[7,9]$ which in our model has the following form:

$$
\begin{aligned}
& \left|\Psi_{\boldsymbol{k}, i}^{(n)}\right\rangle=\sqrt{W_{\boldsymbol{k}, i}^{\mathrm{QP}}}\left[p_{\boldsymbol{k}, i, s}^{\dagger}+\sum_{\boldsymbol{q}_{1}, i_{1}} M_{i i_{1}}\left(k, \boldsymbol{q}_{1}\right) G_{i_{1}}\left(k_{1}, \omega_{1}\right) p_{\boldsymbol{k}_{1, i_{1},-s}}^{\dagger} \beta_{\boldsymbol{q}_{1}}^{\dagger}+\cdots\right. \\
& \quad+\sum_{\boldsymbol{q}_{1} \ldots \boldsymbol{q}_{n}, i_{1} \ldots i_{n}} M_{i i_{1}}\left(k, \boldsymbol{q}_{1}\right) G_{i_{1}}\left(k_{1}, \omega_{1}\right) \cdots \\
& \left.\quad \times M_{i_{n-1} i_{n}}\left(k_{n-1}, \boldsymbol{q}_{n}\right) G_{i_{n}}\left(k_{n}, \omega_{n}\right) p_{k_{n}, i_{n},(-1)_{s}}^{\dagger} \beta_{\boldsymbol{q}_{1}}^{\dagger} \cdots \beta_{\boldsymbol{q}_{n}}^{\dagger}\right]|0\rangle
\end{aligned}
$$

with the momentum $k_{i}=\boldsymbol{k}-\boldsymbol{q}_{1}-\ldots-\boldsymbol{q}_{i}$, and energy $\omega_{i}=\varepsilon_{\boldsymbol{k}}-\omega_{\boldsymbol{q}_{1}}-\ldots-\omega_{\boldsymbol{q}_{i}}$ conservation satisfied by each term. The QP energy is determined by $\varepsilon_{i}(k)=$ $E_{i}(k)+\Sigma_{i}\left(k, \varepsilon_{i}(k)\right)$, and $W_{k, i}^{\mathrm{QP}}$ stands for the QP spectral weight.

The numerical calculations were conducted on $26 \times 26$ and $34 \times 34$ lattices with torroidal boundary conditions. The results were obtained for the realistic parameters of $\mathrm{CuO}_{2}$ planes in high- $T_{\mathrm{c}}$ superconductors, as derived from the electronic structure calculations [10]: $t_{p d}=1.4, U=10$, and $\Delta_{\sigma(\pi)}=5.0$ (all in eV). The spin waves were calculated for the superexchange interaction $J=0.15 \mathrm{eV}[11]$. We also included a realistic $2 p_{\sigma(\pi)}-2 p_{\sigma(\pi)}$ oxygen hopping element $t_{p p}=0.4 \mathrm{eV}$ [2] and assumed $t_{p p}^{\prime}=t_{p p} / 2$ between $2 p_{\sigma(\pi)}-2 p_{\pi(\sigma)}$ neighbouring orbitals.

The coupling of a moving hole to the spin background changes the spectral function in a drastic way. Instead of band-like features due to the oxygen hole which moves only over $2 p$ orbitals (Fig. 1a), one finds nondispersive bound states at low energies (Fig. 1b). The main features seen in the experiment of Pothuizen et al. [2] are well reproduced by the present model calculation (see Fig. 1b). Close to the $(\pi / 2, \pi / 2)$ point the ZR bound states build up with the asymmetry of their intensity around the point $(\pi / 2, \pi / 2)$. Approaching the $(\pi, \pi)$ point we have found $2 p_{\pi}$ coherent band-like states at $\omega \approx-2.4 \mathrm{eV}$ in agreement with the symmetry considerations (see Fig. 3 in Ref. [2]). 

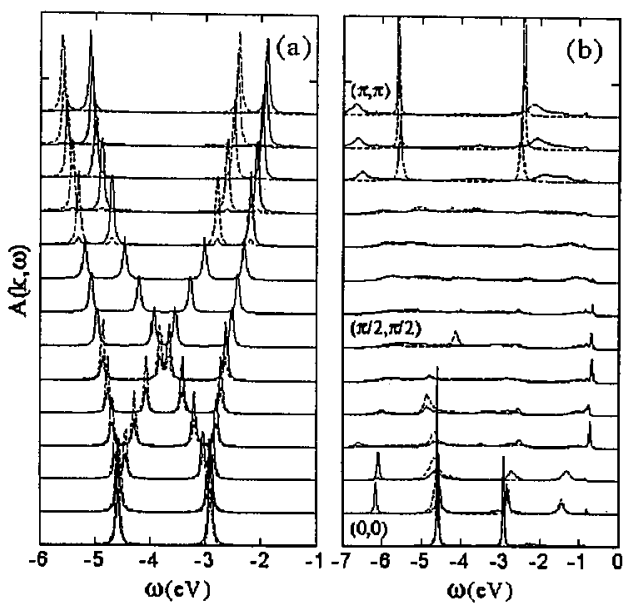

Fig. 1. The electronic spectral functions for (a) the oxygen hole without $\mathrm{Cu}-\mathrm{O}$ interactions (including only direct $2 p-2 p$ hoppings) and (b) in the SCBA for the realistic parameters along the $(0,0)-(\pi, \pi)$ direction. Solid and dashed lines stand for states with $\sigma$ and $\pi$ symmetry, respectively. The broadening (a) $\epsilon=0.03$ and (b) $\epsilon=0.015$ is used.

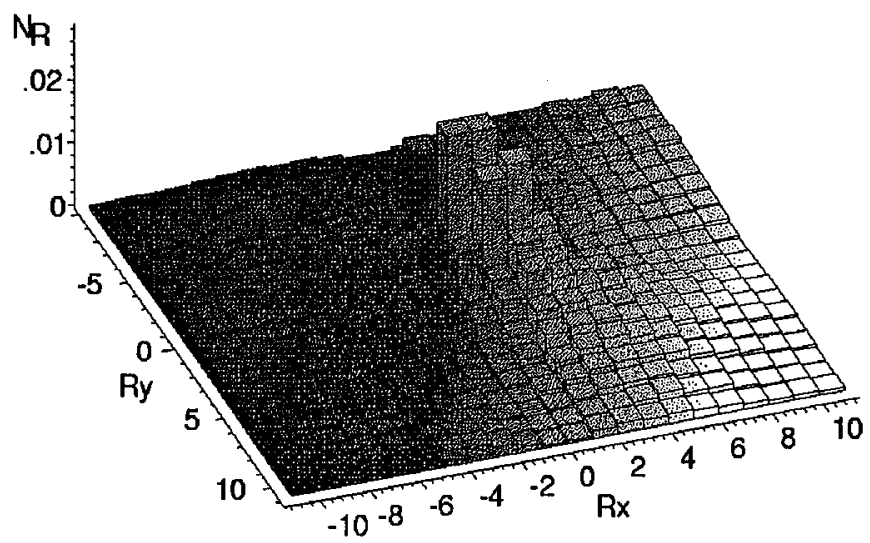

Fig. 2. Spin deviations around the hole, $N_{R}$, calculated at the QP-band minimum at $k=(0.45 \pi, 0.45 \pi)$ for the realistic parameters of $\mathrm{CuO}_{2}$ planes.

In Fig. 2 we display the distribution of bosons around the oxygen hole, $N_{R}=\left\langle n_{0} b_{i \uparrow(\downarrow)}^{\dagger} b_{i \uparrow(\downarrow)}\right\rangle$, where $n_{0}$ is the oxygen hole density operator and $b_{i \uparrow(\downarrow)}$ are bosonic operators for $\mathrm{Cu}$ spins. The spin polaron formed around the hole is extended in the $\langle 11\rangle$ direction of the hole momentum, reflecting the asymmetry of the QP band energy. This asymmetry is similar to that found before for the $t-J$ model [9]. Different spatial structure of the polaron, which extends instead in the $\langle 10\rangle$ and $\langle 01\rangle$ directions, was found for smaller CT elements.

Finally, in Fig. 3 we present the spin-spin correlation function, $C_{R}=$ $\left\langle n_{0}\left(\boldsymbol{S}_{i} \boldsymbol{S}_{j}\right)\right\rangle$, and the bond spin currents, $j_{R}^{z}=\left\langle n_{0}\left(\boldsymbol{S}_{i} \times \boldsymbol{S}_{j}\right)^{z}\right\rangle$, where $\boldsymbol{S}_{i}$ is the copper spin operator. AF correlations are reduced but persist in the vicinity of 

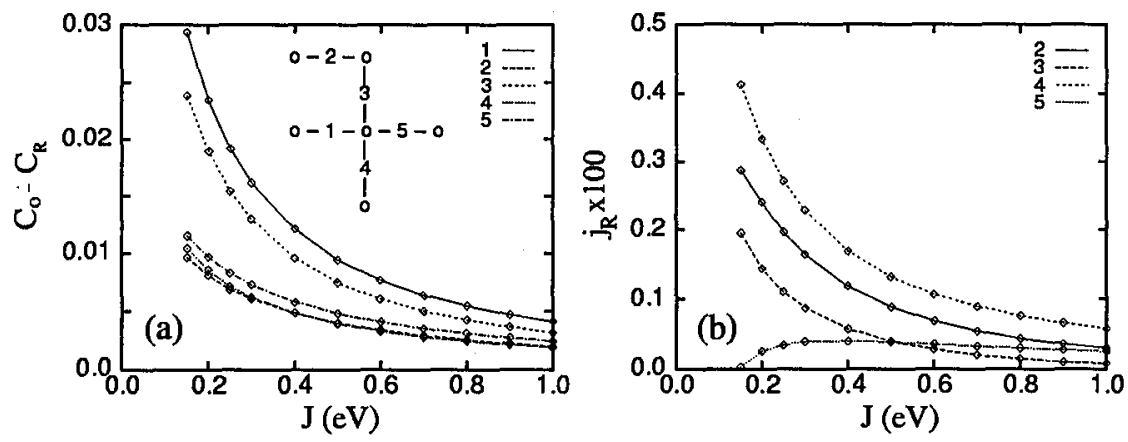

Fig. 3. $C_{R}(\mathrm{a})$ and $j_{R}^{z}$ (b) correlation functions as a function of the superexchange interaction $J$ calculated at the QP-band minimum at $k=(0.45 \pi, 0.45 \pi)$. The inset in (a) shows the labels of $\mathrm{Cu}-\mathrm{Cu}$ bonds for which the data are shown ( 0 are the copper sites). The hole is located at oxygen in the centre of bond 1.

the oxygen hole. Both correlation functions are weaker than in the $t-J$ model due to the oxygen hoppings which diffuse the compensation cloud around the moving hole. In summary, we have shown that our spectra reproduce the experimental results reported for $\mathrm{Sr}_{2} \mathrm{CuO}_{2} \mathrm{Cl}_{2}$. The spin polarons are more extended and characterized by weaker spin fluctuations around the hole than in the $t-J$ model.

We acknowledge the financial support of the Committee for Scientific Research (Poland), project No. 2 P03B 17514.

\section{References}

[1] B.O. Wells, Z.X. Shen, A. Matsuura, D.M. King, M.A. Kastner, M. Greven, R.J. Birgeneau, Phys. Rev. Lett. 74, 964 (1995).

[2] J.J.M. Pothuizen, R. Eder, N.T. Hien, M. Matoba, A.A. Menovsky, G.A. Sawatzky, Phys. Rev. Lett. 78, 717 (1997).

[3] F.C. Zhang, T.M. Rice, Phys. Rev. B 37, 3759 (1988).

[4] A. Nazarenko, K.J.E. Vos, S. Haas, E. Dagotto, R.J. Gooding, Phys. Rev. B 51, 8676 (1995); J. Bała, A.M. Oleś, J. Zaanen, ibid. 52, 4597 (1995).

[5] A. Ramšak, P. Prelovšek, Phys. Rev. B 42, 10415 (1990); O.A. Starykh, O.F. de Alcantara Bonfim, G.F. Reiter, ibid. 52, 12534 (1995); H. Eskes, R. Eder, ibid. 54, 14226 (1996); F. Lema, A.A. Aligia, ibid. 55, 14092 (1997).

[6] J. Bała, A.M. Oleś, J. Zaanen, Phys. Rev. B 54, 10161 (1996).

[7] G.F. Reiter, Phys. Rev. B 49, 1536 (1994).

[8] S. Schmitt-Rink, C.M. Varma, A.E. Ruckenstein, Phys. Rev. Lett. 60, 2793 (1989); C.L. Kane, P.A. Lee, N. Read, Phys. Rev. B 39, 6880 (1989).

[9] A. Ramšak, P. Horsch, Phys. Rev. B 48, 10559 (1993); ibid. 57, 4308 (1998).

[10] J.B. Grant, A.K. McMahan, Phys. Rev. B 46, 8440 (1992).

[11] H. Eskes, J.H. Jefferson, Phys. Rev. B 48, 9788 (1993). 